\title{
Two-Color Two-Photon Excitation Using Femtosecond Laser Pulses
}

\author{
Stefan Quentmeier, ${ }^{\dagger}$ Stefan Denicke, ${ }^{\dagger}$ Jan-Eric Ehlers, ${ }^{\dagger}$ Raluca A. Niesner, ${ }^{\dagger}$ and \\ Karl-Heinz Gericke*, ${ }^{*}$ \\ University of Braunschweig, Institute for Physical und Theoretical Chemistry, Hans-Sommer-Strasse 10, \\ 38106 Braunschweig, Germany, and Helmholz Zentrum für Infektionsforschung, Inhoffenstrasse 7, \\ 38124 Braunschweig, Germany
}

Received: December 3, 2007; In Final Form: January 30, 2008

\begin{abstract}
The use of two-color two-photon (2c2p) excitation easily extends the wavelength range of Ti:sapphire lasers to the UV, widening the scope of its applications especially in biological sciences. We report observation of $2 c 2 p$ excitation fluorescence of $p$-terphenyl (PTP), 2-methyl-5-t-butyl-p-quaterphenyl (DMQ) and tryptophan upon excitation with 400 and $800 \mathrm{~nm}$ wavelengths using the second harmonic and fundamental wavelength of a mode-locked Ti:sapphire femtosecond laser. This excitation is energetically equivalent to a one-photon excitation wavelength at $266 \mathrm{~nm}$. The fluorescence signal is observed only when both wavelengths are spatially and temporally overlapping. Adjustment of the relative delay of the two laser pulses renders a cross correlation curve which is in good agreement with the pulse width of our laser. The fluorescence signal is linearly dependent on the intensity of each of the two colors but quadratically on the total incident illumination power of both colors. In fluorescence microscopy, the use of a combination of intense IR and low-intensity blue light as a substitute for UV light for excitation can have numerous advantages. Additionally, the effect of differently polarized excitation photons relative to each other is demonstrated. This offers information about different transition symmetries and yields deeper insight into the two-photon excitation process.
\end{abstract}

\section{Introduction}

In recent years, two-photon laser scanning microscopy $(2 \mathrm{pm})^{1}$ has become an indispensable tool especially for biological science as the use of more than one photon provides numerous advantages. First, separation of excitation and fluorescence light is much easier because of the larger difference in wavelength compared to conventional confocal microscopy. Therefore better signal-to-noise ratios and high-contrast pictures can be obtained. Second, the usage of IR excitation light instead of UV/vis light leads to reduced photo damage and higher penetration depth ${ }^{2}$ in biological tissue due to lower absorption coefficients in the IR region ${ }^{3}$ than in the visible which is usually used for confocal microscopy. Additionally, due to the quadratic dependence on the intensity, excitation in $2 \mathrm{pm}$ is limited to the focal area only. Thus, out-of-focus excitation is avoided leading to limited photo damage and an intrinsic 3D resolution. Most common excitation sources for $2 \mathrm{pm}$ are Ti:sapphire (Ti:Sa) lasers. The femtosecond (fs) pulses from these lasers are almost ideal for this purpose as they combine high excitation power during the pulse, which is essential for a good excitation yield, ${ }^{4}$ with low-energy deposition to the sample preventing it from being photo damaged. Furthermore, pulsed excitation furnishes the basis for excellent time resolution using time-gated ${ }^{5}$ or time-correlated single-photon-counting (TCSPC) ${ }^{6}$ detection systems. Hence, a variety of time-resolved fluorescence techniques such as fluorescence lifetime imaging (FLIM) ${ }^{5,7-9}$ or fluorescence anisotropy imaging (Tr-FAIM $)^{8}$ have been established.

Typically, $2 \mathrm{pm}$ and other spectroscopic two-photon methods are performed almost exclusively using two excitation photons

\footnotetext{
* Corresponding author. E-mail: k.Gericke@tu-bs.de.

$\dagger$ University of Braunschweig, Institute for Physical und Theoretical Chemistry.

$\doteqdot$ Helmholzzentrum für Infektionsforschung
}

of the same wavelength. Only very few attempts have been made so far to extend these methods to two-color two-photon excitation. ${ }^{10-12}$ This is surprising as the use of two photons at different wavelengths for excitation offers the opportunity to extend the wavelength range where the Ti:Sa laser can be used under retention of all advantages of two-photon excitation. According to theoretical considerations, spatial resolution ${ }^{13}$ and penetration ability, ${ }^{14}$ depending on the microscopical setup, are expected to improve for $2 \mathrm{c} 2 \mathrm{p}$ excitation compared to confocal or conventional $2 \mathrm{pm}$ microscopy, respectively.

Using the fundamental and second harmonic of a Ti:Sa laser, $2 c 2 p$ excitation in the UV can be achieved. As modern modelocked femtosecond lasers offer a tunable range from about 700 to $1000 \mathrm{~nm}$, the conventional $1 \mathrm{c} 2 \mathrm{p}$ technique excites fluorophores in a spectral range of about $350-500 \mathrm{~nm}$. With $2 \mathrm{c} 2 \mathrm{p}$, an additional spectral window from about 230 to $330 \mathrm{~nm}$ is at hand extending the wavelength range of the Ti:Sa laser to the UV where, for example, tryptophan, a fluorescent amino acid responsible for protein fluorescence, ${ }^{15,16}$ can easily be excited. Furthermore, this spectral window corresponds to the excitation wavelength of DNA. ${ }^{17}$

In addition, $2 c 2 p$ excitation offers a unique opportunity for getting a deeper understanding of two-photon absorption processes. Here, in contrast to $1 \mathrm{c} 2 \mathrm{p}$ experiments, it is possible to manipulate the polarization of each of the absorbed photons independently. Theoretical considerations $s^{9,18}$ as well as $2 c 2 p$ experiments conducted as absorption measurements ${ }^{19}$ imply that there is a close relationship between the relative polarizations of the two photons and the symmetry of the involved electronic states. The absorption data published by McClain ${ }^{18,19}$ lead to the assumption that it should be possible to discriminate chromophores possessing different symmetries by their different behavior toward different excitation polarizations. An additional 


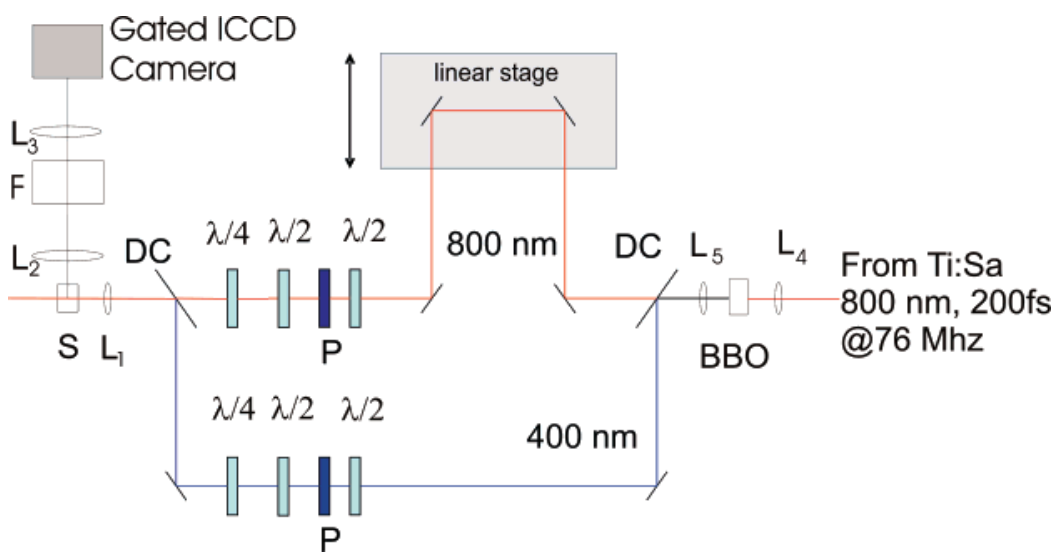

Figure 1. Experimental setup. $\mathrm{BBO}=$ barium borate crystal for frequency doubling, $L=$ lens, $\mathrm{DC}=$ dichroic mirror, $\lambda / 2=$ half wave plate, $\lambda / 4$ $=$ quarter wave plate, $F=$ set of filters (Semrock NF01-405U, Semrock NF01-808U both at $10^{\circ}$ angle, Schott UG 11 and a interference filter $340 / 80 \mathrm{~nm})$.

indication for this assumption is given by the work of Lakowicz ${ }^{10}$ et al. where a different behavior of PTP and indole toward the excitation with perpendicularly polarized photons was found. The effect of circularly polarized photons is still unclear.

To the best of our knowledge, we present the first observation of $2 \mathrm{c} 2 \mathrm{p}$ excitation fluorescence using a modern passive modelocking Ti:Sa laser source emitting femtosecond pulses. The fundamental and second harmonic of a Ti:Sa laser are focused confocally through a lens into a cuvette where fluorescence is observed as a result of $2 \mathrm{c} 2 \mathrm{p}$ excitation. The phenomenon is observed for PTP, DMQ, and tryptophan.

Furthermore, we present the first fluorescence lifetime measurements performed in the time domain studying $2 c 2 p$ excitation fluorescence and, in addition, the behavior for different linearly and circularly polarized photons.

\section{Material and Methods}

The femtosecond laser pulses are provided by a Ti:Sa laser (Mira 900B, Coherent) which is pumped by a Nd:YVO $\mathrm{YV}_{4}$ solidstate laser (Verdi V10, coherent). The Ti:Sa is operated at 800 $\mathrm{nm}$ with a pulse width of $200 \mathrm{fs}$. The initial $800 \mathrm{~nm}$ beam is frequency doubled using a $\beta$-barium borate (BBO) crystal (Figure 1). The two colors, that is, the frequency-doubled and the remaining fundamental light, are then separated by a dichroic mirror so that blue and red light can be manipulated independently in terms of power, polarization, and optical path length. Power adjustment is realized using a set of a $\lambda / 2$ plate (all wave plates: quartz, low-order, coated) and a thin layer polarizer (P) for each beam. All states of linear and circular polarization can be prepared for each color separately using a set of $\lambda / 2$ and $\lambda / 4$ plates. The degree of polarization for linear and circular cases are measured using an analyzer and a power meter for the linear case and a set of Fresnel Rhomb, polarizer, and power meter for the circular case, respectively. All degrees of polarization used for the measurements are in the range of $95-99 \%$. The optical path length of the $800 \mathrm{~nm}$ light can be adjusted using a delay line in the red path consisting of two mirrors mounted to a precise linear stage to provide temporal overlap (Figure 2).

After reunion of the two colors by a second dichroic mirror, both beams are focused in a confocal setup through an achromatic lens with a $5 \mathrm{~cm}$ focal length into the sample located in a $5 \times 5 \mathrm{~mm}^{2}$ cuvette. For adjustment, this cuvette can be replaced by a pinhole or a second BBO crystal for accomplishing spatial and temporal overlap, respectively. Temporal overlap is

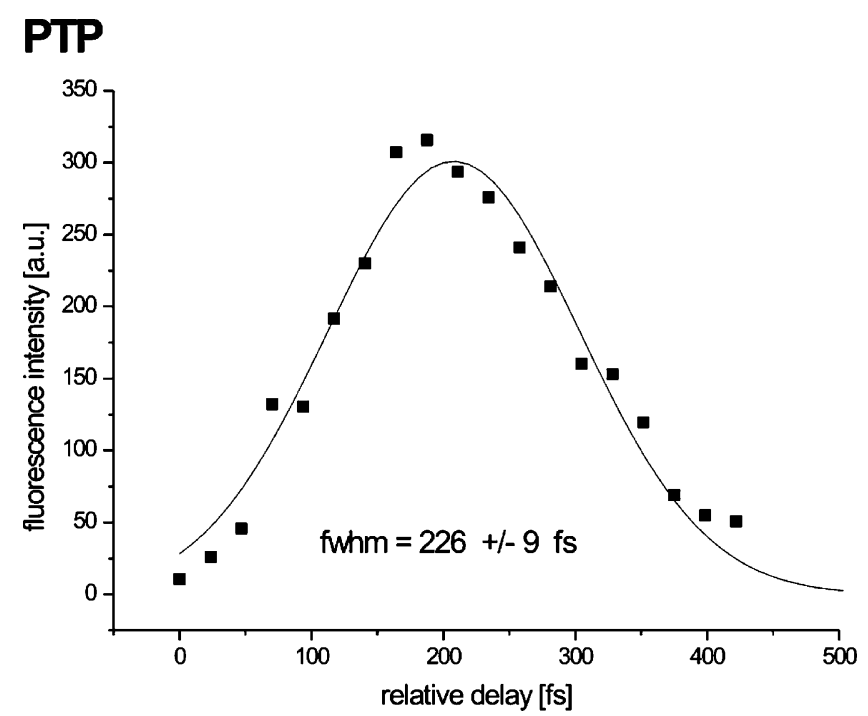

Figure 2. Cross correlation curve showing the dependence of the fluorescence intensity on the temporal overlap. The resulting fwhm value is in good agreement with the pulse width of the laser.

indicated by wave mixing of the 800 and $400 \mathrm{~nm}$ beams resulting in $266 \mathrm{~nm}$ emission from the crystal.

Fluorescence is monitored in a perpendicular setup through a set of filters consisting of UG 11 (Schott), a 340/80 nm interference filter, and two 400 and $800 \mathrm{~nm}$ notch filters (Semrock) and detected by a time-gated ICCD camera system (picostar, LaVision GmbH, Göttingen). The gate width of the camera system can be adjusted from $200 \mathrm{ps}$ to $1 \mathrm{~ns}$ and, in combination with a delay generator (Kentech Instruments Ltd.), a time resolution as good as 10 ps can be achieved. ${ }^{20}$

PTP and DMQ were purchased from Lamda Physik AG, Germany, and used without further purification. PTP and DMQ were dissolved in cyclohexane to give $1 \mathrm{mM}$ solutions. Tryptophan was purchased from Sigma-Aldrich to prepare a 1 $\mathrm{mM}$ solution in PBS buffer.

\section{Results}

Two-color two-photon fluorescence was studied for different dyes. First, observations were made for PTP in order to have comparable results with respect to previous publications ${ }^{11}$ using picosecond dye lasers. After this comparison, we studied other dyes where $2 c 2 p$ fluorescence is unknown. We found clear evidence for $2 \mathrm{c} 2 \mathrm{p}$ excitation to be the origin of the detected 

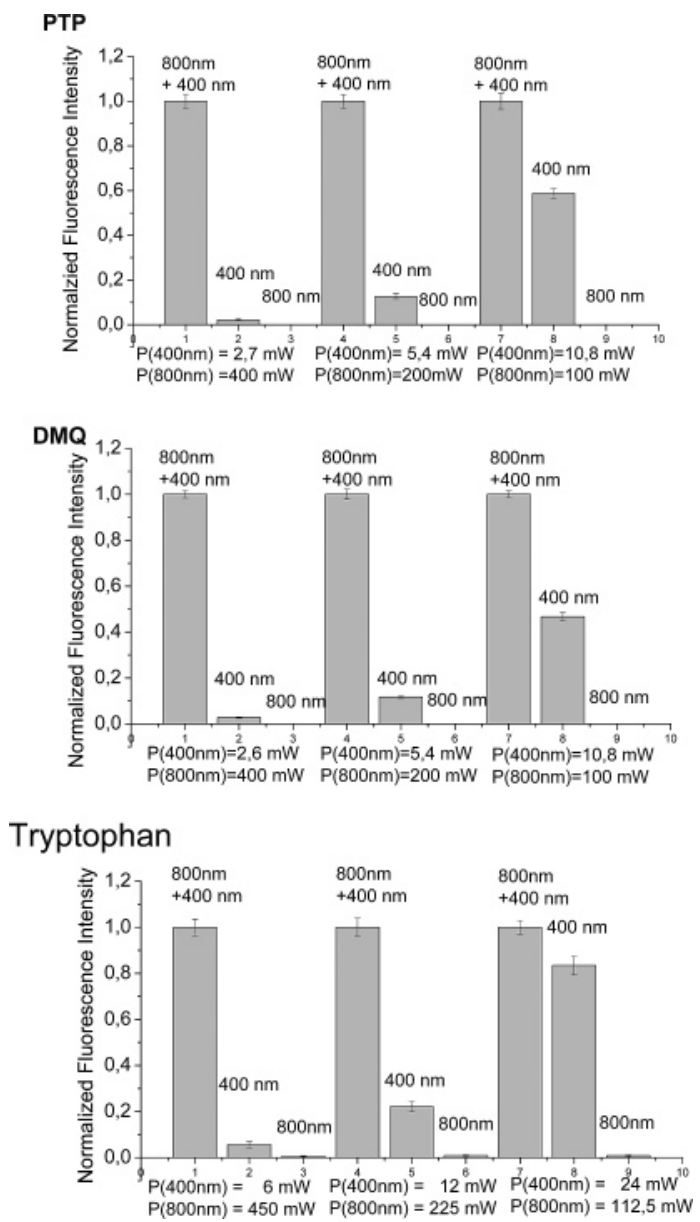

Figure 3. Fluorescence intensity for irradiation by one or two laser beams at different power ratios of the 400 and $800 \mathrm{~nm}$ light. Highest fluorescence intensities are observed for irradiation with both colors. Alone, $400 \mathrm{~nm}$ produces a fluorescence signal from $1 \mathrm{c} 2 \mathrm{p}$ excitation. This can be suppressed by using less $400 \mathrm{~nm}$ light and more $800 \mathrm{~nm}$ light, as $800 \mathrm{~nm}$ light alone produces no signal even at high intensities. Each data point represents the mean value of 20-30 measurements, and the error bars show the standard deviation.

fluorescence of PTP, DMQ, and tryptophan. The most straightforward observation is that the full fluorescence signal is present only when the sample is illuminated by both laser beams and only when they temporarily overlap.

As shown in Figure 3, strong fluorescence is observed only when both beams are irradiated into the sample. The columns labeled $800 \mathrm{~nm}+400 \mathrm{~nm}$ represent the $2 \mathrm{c} 2 \mathrm{p}$ fluorescence signal which is obtained by subtracting the signals for each color alone from the signal which is present when both beams are irradiated. For better comparison the data were normalized to the $2 c 2 p$ signal. Using $400 \mathrm{~nm}$ exclusively, we observe a weak fluorescence signal. Further investigations of the fluorescence from the blue excitation light alone showed a quadratic dependence of the fluorescence intensity on the excitation power (Figure 4).

This indicates a one-color two-photon (1c2p) absorption being the origin of that fluorescence. This assumption is in good agreement with the one-photon absorption spectra ${ }^{21,22}$ which all show a strong second absorption band around $200 \mathrm{~nm}$. However, in all cases, almost no signal is detected for irradiation of 800 nm only. As can be seen from Figure 3, attenuation of the blue beam with simultaneous increase of the power of the red beam easily allows suppressing the undesired 1c2p signal with retention of the $2 c 2 p$ fluorescence intensity. This is possible because in the case of a two-photon absorption the absorption
PTP
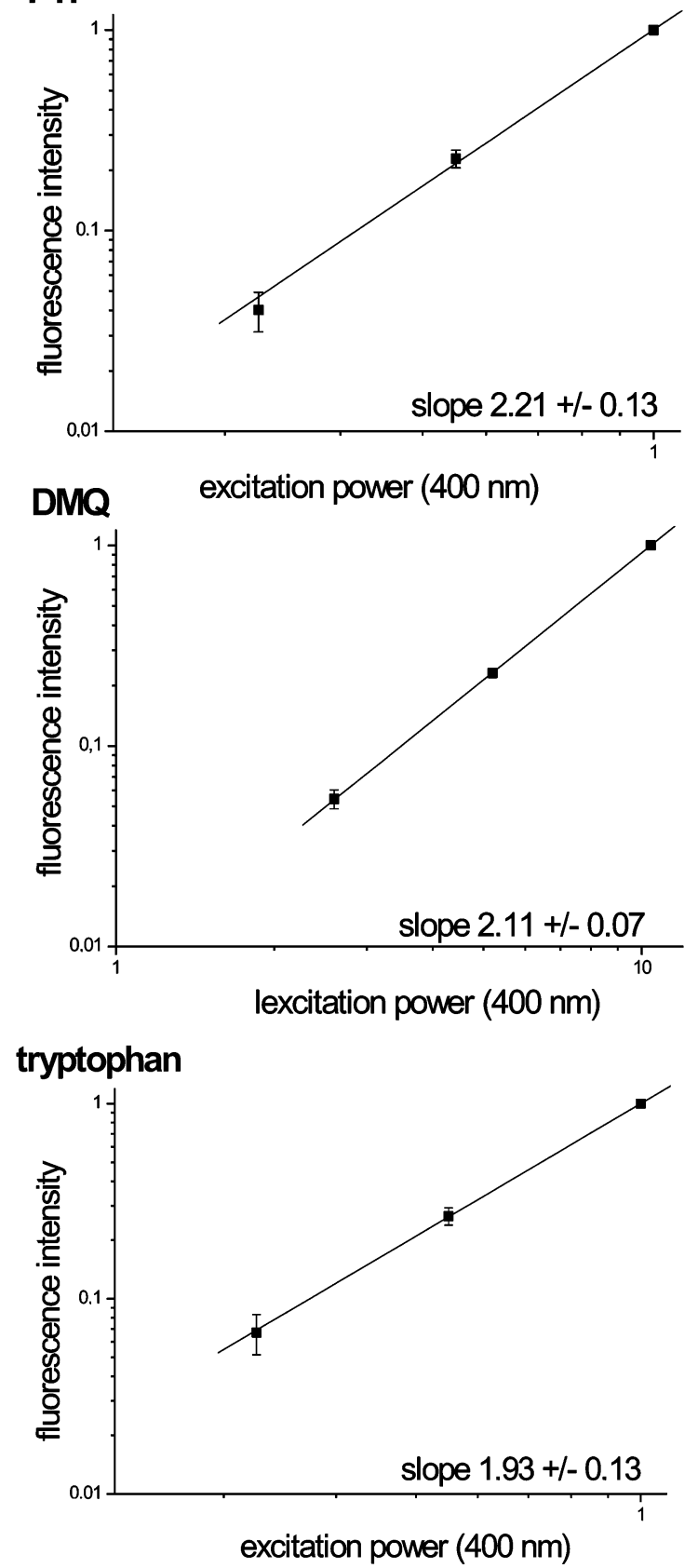

Figure 4. Dependence of the fluorescence intensity on the excitation power when only $400 \mathrm{~nm}$ light is irradiated. The quadratic dependence indicates a one-color two-photon excitation (1c2p).

rate is linearly dependent on the product of the intensities of each color.

$$
\frac{\mathrm{d} N}{\mathrm{~d} t}=-\delta I_{800 \mathrm{~nm}} I_{400 \mathrm{~nm}} N
$$

where $N=$ number of molecules in the ground state, $\delta=$ twophoton absorption cross section, and $I=$ photon flux.

Therefore the fluorescence intensity is expected to be linearly dependent on each of the excitation intensities providing the other is held constant. If the power of the $400 \mathrm{~nm}$ beam is attenuated, the $2 \mathrm{c} 2 \mathrm{p}$ signal will decrease proportionally while the $1 c 2 p$ signal will decrease more rapidly because of its quadratic dependence. If the power of both colors is adjusted, one expects a quadratic dependence on the total power of both colors. The data shown in Figure 5 indicate that our experimental results are in very good agreement with these considerations. 

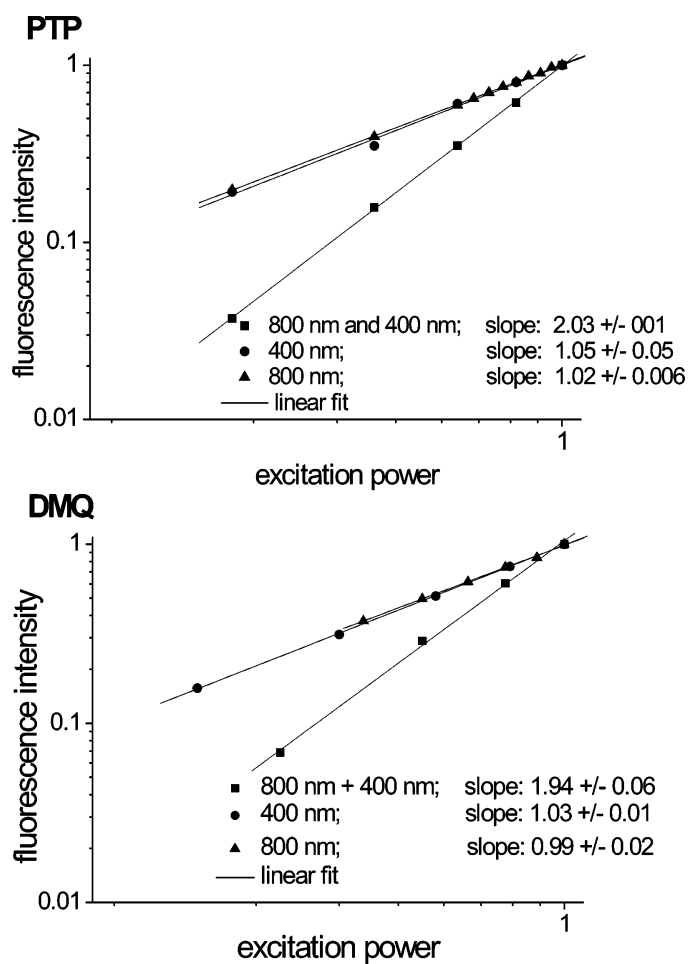

Figure 5. Dependence of $2 c 2 p$ fluorescence intensity on variation of the power level of one or both colors. If the power of only one color is changed, a linear dependence is found. For simultaneous variation of the power of both colors a quadratic dependence on the total excitation intensity is found.

For all dyes and different power levels, a three-photon excitation is not observed although for $800 \mathrm{~nm}$ irradiation this would be energetically equivalent to a $2 c 2 p$ excitation with 800 and $400 \mathrm{~nm}$. It seems that much higher intensities would be necessary because of the extremely small three-photon absorption cross sections.

Another confirmation for the presence of $2 c 2 p$ excitation is a cross correlation experiment where the time delay between both laser pulses is varied. As both photons are required for the $2 c 2 p$ excitation, the maximal fluorescence intensity is expected only when both laser pulses are exactly temporally overlapping. As expected, this experiment renders a cross correlation curve (Figure 2) whose fwhm value of $226 \pm 9$ fs is in good agreement with the pulse width of the Ti:Sa laser in this experiment.

The pulse width of the $800 \mathrm{~nm}$ light was measured after the second dichroic mirror rendering an autocorrelation curve with an fwhm value of 260 fs. Assuming a Gaussian pulse shape, this corresponds to a pulse width of $(260 \mathrm{fs}) / \sqrt{ } 2=184 \mathrm{fs}$. Hence the pulse width of the blue light can be expected to have a pulse width of $(184 \mathrm{fs}) / \sqrt{ } 2=130$ fs for a quadratic dependence of the SHG on the $800 \mathrm{~nm}$ power. Therefore, the expected value for a cross correlation of these two pulses is $\sqrt{\left((130 \mathrm{fs})^{2}+(184 \mathrm{fs})^{2}\right)}=225 \mathrm{fs}$ endorsing the measured value of $226 \pm 9$ fs.

To confirm that the $2 \mathrm{c} 2 \mathrm{p}$ excitation leads to fluorescence emission from the $S_{1}$ state, fluorescence lifetimes were measured (Figure 6) using a time-gated intensified camera system.

All observed lifetimes are in good agreement with those known from literature ${ }^{23-25}$ for a one-photon excitation. Additionally, we found no difference in the fluorescence lifetime for $1 \mathrm{c} 2 \mathrm{p}$ or $2 \mathrm{c} 2 \mathrm{p}$ excitation. Thus, it can be concluded that the origin of the fluorescence is the same electronic state, that is, the $\mathrm{S}_{1}$ state.
The effect of differently polarized excitation photons on the fluorescence intensity is also investigated. As shown in Figure 7, a change from two parallel polarized photons ( $\mathrm{r}$ ls b ls) to two perpendicular photons ( $\mathrm{r} 1 \mathrm{~s} \mathrm{~b} \mathrm{lp}$ ) results in a reduction of fluorescence intensity to approximately $40 \%$ of the parallel case. For excitation with two circular polarized photons ( $\mathrm{r} \mathrm{cr} \mathrm{b} \mathrm{cl}$ and $\mathrm{r} \mathrm{cr} \mathrm{b} \mathrm{cr}$ ) we also find reduced fluorescence intensities. As can be seen from Figure 7, the intensities resulting from circular polarization are higher than those from linear polarized photons being oriented perpendicular to each other but still smaller than from the parallel case. We could not find a significant difference for circular polarized photons with same ( $\mathrm{r} \mathrm{cr} \mathrm{b} \mathrm{cr)} \mathrm{or} \mathrm{opposite}$ $(\mathrm{r} \mathrm{cr} \mathrm{b} \mathrm{cl)} \mathrm{sense} \mathrm{of} \mathrm{rotation.} \mathrm{The} \mathrm{differences} \mathrm{in} \mathrm{fluorescence}$ intensities are influenced by the symmetry of all involved electronic states. In the case of a two-photon absorption, this means that the virtual states have a contribution to this effect, too. A detailed theory allowing conclusions from the measured polarization dependency on the symmetry of the molecule requires advanced theoretical analysis which needs to be done.

\section{Discussion}

Two-color two-photon excitation is a powerful tool for enlarging the spectral range of nowadays femtosecond $\mathrm{Ti}: \mathrm{Sa}$ laser systems being used for spectroscopy and two-photon microscopy. We demonstrate measurements of $2 c 2 p$ excitation fluorescence using femtosecond pulses from a Ti:Sa laser. Since these experiments were carried out using a time-gated camera system as a detector which is normally used for fluorescence lifetime imaging, we believe that applications of $2 c 2 p$ excitation to microscopy are now within reach. The use of femtosecond pulses instead of picosecond pulses has several advantages, although the securing of a temporal overlap is definitely more challenging the shorter the pulses become. For multiphoton processes, high excitation powers are desired as the rate of absorption is dependent on the square of the total photon flux used for excitation in combination with very small multiphoton absorption cross sections. On the other hand, the amount of energy introduced into the sample should be as low as possible to avoid damage. The solution for this dilemma is the use of pulses as short as possible. ${ }^{4}$ The ideal light source for ultra short pulses today is the Ti:Sa laser emitting femtosecond pulses in high repetition and high power output. Our experiments show that even with femtosecond pulses it is possible to have stable spatial and temporal overlap in an experimental setup. Furthermore, it is worth mentioning that the implementation of $2 c 2 p$ excitation into a microscope will lead to a much higher photon efficiency. First, the higher focusing of a high numeric aperture objective will result in increased photon fluxes in the focal region. As the excitation rate is quadratically dependent on the photon flux, this will lead to a much more efficient excitation. Second, a higher fraction of the occurring fluorescence photons is collected again, due to the high numeric aperture. Considering both effects, one can conclude that observation of $2 c 2 p$ excitation fluorescence in a microscope will be about 2 orders of magnitude more efficient than in the present experimental setup. In other words, for achieving the same signal intensity only about one percent of the excitation power is required.

The results of $2 c 2 p$ excitation of PTP were in good agreement with those from previous publications where measurements have been performed using cavity-dumped picosecond dye lasers. ${ }^{11}$ In addition, with DMQ and tryptophan we found two other dyes suitable for $2 c 2 p$ excitation using 800 and $400 \mathrm{~nm}$ for excitation which have not been studied with $2 \mathrm{c} 2 \mathrm{p}$ excitation yet. All fluorophores show good $2 \mathrm{c} 2 \mathrm{p}$ excitation fluorescence. They also 

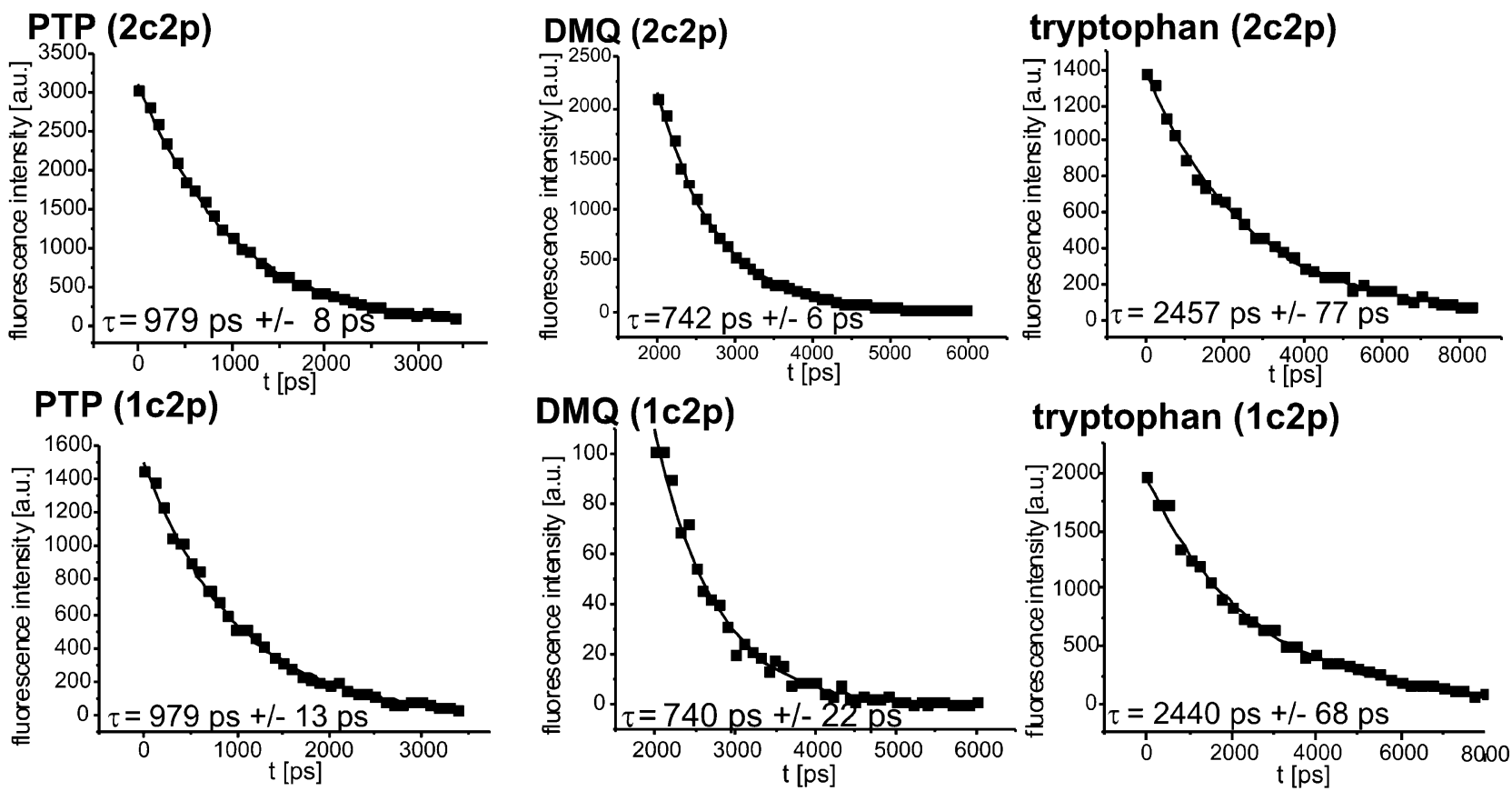

Figure 6. Fluorescence lifetimes of PTP, DMQ, and tryptophan for $2 c 2 p$ and $1 c 2 p$ excitation. For both modes of excitation, similar lifetimes are found indicating that fluorescence emission occurs from the same excited state for both types of excitation. A mono exponential decay is used for fitting the data.

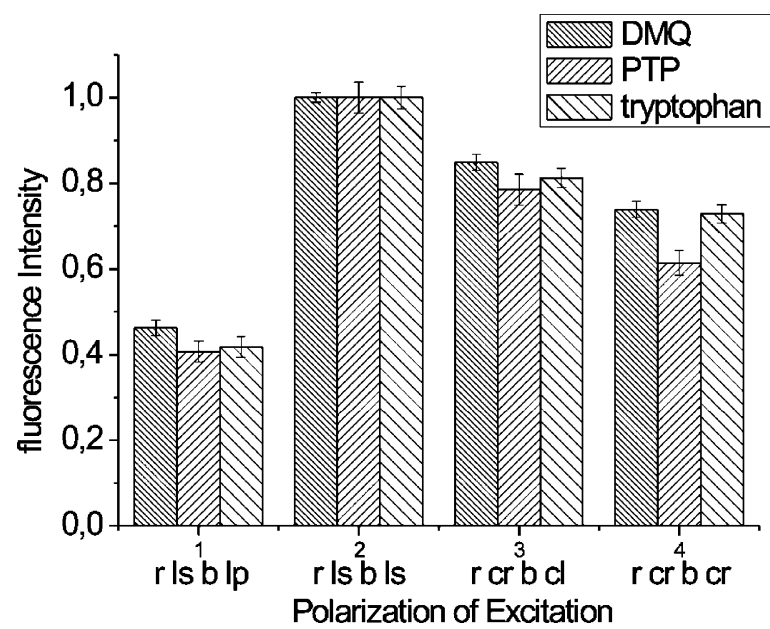

Figure 7. Influence of the relative polarization of the exciting photons on the fluorescence intensity. Two linearly polarized photons which are parallel lead to maximum fluorescence, whereas two linearly polarized photons with an angle of $90^{\circ}$ between the polarizations lead to the weakest fluorescence signal. Circularly polarized photons lead also to a reduced fluorescence signal compared to two parallel photons. $r=$ red light, $b=$ blue light, $1 \mathrm{~s}=$ linear perpendicular polarized, $1 \mathrm{p}=$ linear parallel polarized, $\mathrm{cr}=$ circular polarized with a right sense of rotation, and $\mathrm{cl}=$ circular polarized with a left sense of polarization.

show some $1 \mathrm{c} 2 \mathrm{p}$ excitation fluorescence; however, this occurs only when strong blue light is applied. Therefore it seems crucial to use an excess of red light with less of the blue component. What on first sight looks like a disadvantage turns out to be concurrent with one of the big advantages of $2 c 2 p$ excitation. In fact, for most applications it will be highly desired to use only low-level $400 \mathrm{~nm}$ light to keep photo damage low. The fact that the fluorescence intensity is linearly dependent on the product of the intensities of both colors offers the possibility of maintaining a high penetration depth in combination with low photo damage compared to one-photon UV excitation. The low power of the blue light can easily be compensated for by increasing the power of the IR light which is much less harmful to biological samples. The IR can easily penetrate through biological tissue without being absorbed, and therefore it will not inflict much damage, as experiences with common $2 \mathrm{pm}$ show.

To study the proposed influence of different relative polarizations on the excitation of molecules with different symmetries it is crucial to have a good variety of different dyes.

So far, we found no evidence for such an influence for the studied molecules. The reason might be the similar symmetry of the involved transitions. Future time-and polarization-resolved measurements will render more detailed information on the anisotropic character of the fluorescence decay. So one can expect that two photons being not parallel polarized, as in the case for $1 c 2 p$ excitation, will give a significantly different photo selection. This will influence the observed initial anisotropy after excitation.

Besides these theoretical considerations, the demonstration of tryptophan as a suitable fluorophore for $2 \mathrm{c} 2 \mathrm{p}$ excitation will offer a good variety of possible applications. Tryptophan can be found in many different proteins, and $2 c 2 p$ excitation will allow it to be used as a natural fluorescent tag as well as a fluorescent probe inside the protein without applying UV to proteins. Hence, the advantages of label-free measurements and high time resolution resulting from pulsed excitation with the Ti:Sa will be combined and additionally enriched by the fact that direct UV irradiation is avoided and therefore photo damage can be reduced. Although $2 \mathrm{c} 2 \mathrm{p}$ fluorescence from tryptophan is roughly 20 times weaker than from the other dyes, it is still sufficient for fluorescence analysis. Experiments concerning protein bioassays are in progress.

\section{Conclusion}

We demonstrated that $2 \mathrm{c} 2 \mathrm{p}$ excitation with femtosecond lasers is an option for extending their spectral range in combination with advantages arising from the use of multiphoton excitation. The ultra short femtosecond pulses are an ideal basis for later application in microscopy. Within the newly gained spectral range, tryptophan can be $2 \mathrm{c} 2 \mathrm{p}$-excited and used as a fluorophore. 
Acknowledgment. This work was supported by the Bundesministerium für Bildung und Forschung (BMBF) under Grant $0313412 \mathrm{C}$.

\section{References and Notes}

(1) Denk, W.; Strickler, J. H.; Webb, W. W. Two-Photon Laser Scanning Fluorescence Microscopy. Sci. News Ser. 1990, 248 (4951), 7376.

(2) Niesner, R.; Andresen, V.; Neuman, J.; Spieker, H.; Gunzer, M. The Power of Single and Multibeam Two-Photon Microscopy for HighResolution and High-Speed Deep Tissue and Intravital Imaging. Biophys. J. 2007, 93, 2519-2529.

(3) Weissleder, R.; Ntziachristos, V. Shedding Light onto Live Molecular Targets. Nat. Med. 2003, 9 (1), 123-128.

(4) Niesner, R.; Roth, W.; Gericke, K.-H. Photophysical Aspects of Single-Molecule Detection by Two-Photon Excitation with Consideration of Sequential Pulsed Illumination. ChemPhysChem. 2004, 5, 678-687.

(5) Elson, D.; Requejo-Isidro, J.; Munro, I.; Reavell, F.; Siegel, J.; Suhling, K.; Tadrous, P.; Benninger, R.; Lanigan, P.; McGinty, J.; Talbot, C.; Treanor, B.; Webb, S.; Sandison, A.; Wallace, A.; Davis, D.; Lever, J.; Neil, M.; Phillips, D.; Stamp, G.; French, P. Time-domain fluorescence lifetime imaging applied to biological tissue. Photochem. Photobiol. Sci. 2004, 3, 795-801.

(6) Becker, W.; Bergmann, A.; Biscotti, G.; Rück, A. Advanced timecorrelated single-photon counting technique for spectroscopy and imaging in biomedical systems. Proc. SPIE 2004, 5340, 1-9.

(7) Gratton, E.; Breusegem, S.; Sutin, J.; Ruan, Q.; Barry, N. Fluorescence lifetime imaging for the two-photon microscope: Time-domain and frequency-domain methods. Biomed. Opt. 2003, 8 (3), 381-390.

(8) Suhling, K.; French, P. M. W.; Phillips, D. Time-resolved fluorescence microscopy. Photochem. Photobiol. Sci. 2005, 4, 13-22.

(9) McClain, W. M. Polarization of two-photon excited fluorescence. J. Chem. Phys. 1972, 58 (1), 324-326.

(10) Gryczynski, I.; Malak, H.; Lakowicz, J. R. Two-Color Two-Photon Excitation of Indole. Biospectroscopy 1997, 3 (2), 97-101.

(11) Lakowicz, J. R.; Gryczynsiki, I.; Malak, H.; Gryczynski, Z. TwoColor Two-Photon Excitation of Fluorescence. Photochem. Photobiol. 1996, 64 (4), 632-635.

(12) Palero, J.; Garcia, W.; Saloma, C. Two-color (two-photon) excitation fluorescence with two confocal beams and a Raman shifter. Opt. Commun. 2002, 211, 65-71.
(13) Chen, J.; Midorikawa, K. Two-color two-photon 4Pi fluorescence microscopy. Opt. Lett. 2004, 29 (12), 1354.

(14) Cambaliza, M. O.; Saloma, C. Advantages of two-color excitation fuorescence microscopy with two confocal excitation beams. Opt. Commun. 2000, 184, 25-35.

(15) Lakowicz, J. R.; Gryczynski, I. Tryptophan fluorescence intensity and anisotropy decays of human serum albumin resulting from one-photon and two-photon excitation. Biophys. Chem. 1992, 45, $1-6$.

(16) Fleming, G. R.; Morris, J. M.; Robbins, R. J.; Woolfe, G. J.; Thistlethwaite, P. J.; Robinson, G. W. Nonexponential fluorescence decay of aqueous tryptophan and two related peptides by picosecond spectroscopy. Proc. Natl. Acad. Sci. U.S.A. 1978, 75 (10), 4652-4656.

(17) Pisarevskii, A. N.; Cherenkevich, S. N.; Andrianov, V. T. Fluorescence Spectrum and Quantum Yield of DNA in Solution. Zh. Prikl. Spektrosk. 1966, 5 (5), 621-624.

(18) Monson, P. R.; McClain, W. M. Polarization Dependence of the Two-Photon Absorption of Tumbling Molecules with Application to Liquid 1-Chloronaphthalene and Benzene. J. Chem. Phys. 1969, 53 (1), 29.

(19) Monson, P. R.; McClain, W. M. Complete Polarization Study of the Two-Photon Absorption of Liquid 1-Chloronaphthalene. J. Chem. Phys. 1971, 56 (10), 4817-4825.

(20) Niesner, R.; Peker, B.; Schlüsche, P.; Gericke, K.-H. Noniterative Biexponential Fluorescence Lifetime Imaging in the Investigation of Cellula Metabolism by Means of NAD $(\mathrm{P}) \mathrm{H}$ Autofluorescence. ChemPhysChem 2004, 5, 1141-1149.

(21) Lakowicz, J. R. Principles of Fluorescence Spectroscopy, 3rd ed.; Kluwer Academic/Plenum Publishers: New York, 1999.

(22) Brackmann, U. Lambdachrome Laser Dyes. Lambda Physik GmbH: Göttingen, Germany, 1986.

(23) Wijnaendts van Resand, R.; Vogel, R.; Provencher, S. Doublebeam fluorescence lifetime spectrometer with subnanosecond resolution. Rev. Sci. Instrum. 1982, 53, 1392-1397.

(24) Georghiou, S.; Thompson, M.; Muikhopadhyay, A. K. MelittinPhospholipid Interaction Studied by Employing the Single-Tryptophan Residue as an Intrinsic Fluorescent Probe. Biochim. Biophys. Acta 1982, 688, 441-452.

(25) Güsten, H.; Rinke, M.; Wirth, H. O. Photophysical Properties and Laser Performance of Photostable UV Laser Dyes. Appl. Phys. 1988, 45 $279-284$ 